\title{
Enhancing Video Accessibility and Availability Using Information-Bound References
}

\author{
Ashok Anand \\ Instart Logic \\ ashok.anand@gmail.com \\ Ath \\ Stony Brook University \\ vyas.sekar@gmail.com
}

\author{
Athula Balachandran \\ Carnegie Mellon University \\ abalacha@cs.cmu.edu
}

\author{
Aditya Akella \\ University of Wisconsin, Madison \\ akella@cs.wisc.edu
}

\begin{abstract}
Users are often frustrated when they cannot view video links shared via blogs, social networks, and shared bookmark sites on their devices or suffer performance and usability problems when doing so. While other versions of the same content better suited to their device and network constraints may be available on other third-party hosting sites, these remain unusable because users cannot efficiently discover these and verify that these variants match the content publisher's original intent. Our vision is to enable consumers to leverage verifiable alternatives from different hosting sites that are best suited to their constraints to deliver a high quality of experience and enable content publishers to reach a wide audience with diverse operating conditions with minimal upfront costs. To this end, we make a case for informationbound references or IBRs that bind references to video content to the underlying information that a publisher wants to convey, decoupled from details such as protocols, hosts, file names, or the underlying bits. This paper addresses key challenges in the design and implementation of IBR generation and resolution mechanisms, and presents an evaluation of the benefits IBRs offer.
\end{abstract}

\section{Categories and Subject Descriptors}

H.3.4 [Information storage and retrieval]: Systems and software

\section{Keywords}

video; references; QoE; availability; accessibility

\section{INTRODUCTION}

A significant and growing fraction of Internet traffic today consists of video content [3]. Many users discover and access such content via links shared via traditional (e.g., email, IM) and social media applications (e.g., online social networks,

Permission to make digital or hard copies of all or part of this work for personal or classroom use is granted without fee provided that copies are not made or distributed for profit or commercial advantage and that copies bear this notice and the full citation on the first page. Copyrights for components of this work owned by others than ACM must be honored. Abstracting with credit is permitted. To copy otherwise, or republish, to post on servers or to redistribute to lists, requires prior specific permission and/or a fee. Request permissions from permissions@ acm.org.

CoNEXT'13, December 9-12, 2013, Santa Barbara, California, USA.

Copyright 2013 ACM 978-1-4503-2101-3/13/12 ...\$15.00.

http://dx.doi.org/10.1145/2535372.2535393.

\author{
Srinivasan Seshan \\ Carnegie Mellon University \\ srini@cs.cmu.edu
}

blogging services, and social bookmarking sites [33, 7]). Unfortunately, the URLs used to share videos are fragile as they are inherently bound to a specific protocol, host, and file name [29]. This tight coupling is especially problematic as users want to access content from an increasingly heterogeneous set of device (e.g., smartphones, tablets) and network (e.g., 3G, 4G, WiFi) conditions. As shown in $\S 2$, this results in significant accessibility (e.g., content not playable) and quality-of-experience (QoE) problems (e.g., high buffering or start-up latencies for video [28]).

Fortunately, measurement studies show that there are alternative versions of the same content with different resolutions or formats available on different third-party hosting sites $[2,32]$. We could potentially alleviate accessibility and QoE issues if we had a mechanism that enables users to find the alternative best suited to their current software, device, and network context. A variety of entities, including device vendors, search engine providers, and social networking and bookmarking sites would have strong incentives to deploy such a mechanism $(\S 2)$.

However, it is challenging to realize such a mechanism in practice. To see why, consider two seemingly natural strawman approaches. To delay the binding to a specific video file, one option for content publishers is to only provide keywords. Content consumers can then use search engines to find suitable alternatives. Unfortunately, search keywords are notoriously contention prone and give users no confidence that the result matches the publisher's intent [29]. Alternatively, one could envision new data-centric architectures as they decouple the data from the delivery mechanisms [26, 38]. The hash-based naming schemes in these proposals, however, operate at the byte-level. Thus, the names for different encodings of the same content will be different and preclude opportunities for leveraging the alternative versions.

The key here is choosing an appropriate granularity at which we need to bind the intent of the publisher. Datacentric names offer one extreme at the byte-level representation. Human-readable keywords offer another point with loose bindings that are susceptible to abuse. What we ideally need is a mechanism that delays the binding sufficiently to provide the flexibility to choose alternatives, but at the same time allows clients to be assured that the variant they choose matches the publishers' original intent. We call this new type of link an Information-Bound Reference or IBR since it references the information that a user wants rather than its location, format, or file name. Using IBRs will 
allow content publishers to reach a broader audience without significant quality problems and without high upfront infrastructure costs. IBRs will improve consumers' quality of experience and minimize frustrations due to poor performance or content inaccessibility.

In this paper, we address key algorithmic and system design challenges in realizing an IBR-based content retrieval framework. Our key contributions are:

- IBR Generation: IBRs must be encoding-invariant, resilient to contention (i.e., two unique pieces of information map to different IBRs) and compact (i.e., be small relative to the content they refer to). To this end, we envision a novel use-case for multimedia fingerprinting algorithms [35, 34, 19]. Our vision, however, raises new performance, scalability, and verifiability requirements and thus our specific contribution lies in practically synthesizing these techniques to serve as IBRs $(\S 4)$.

- Resolution: Users should be able to use IBRs to quickly find the copy of content that is most appropriate for their device and network conditions. What makes this challenging is that video encodings are intrinsically lossy which means that the IBR matching involves a "fuzzy" match. To this end, we design a scalable lookup service that can provide $\approx 1$ million lookups/s on a 25 -node cluster leveraging algorithms for locality-sensitive hashing [24] (§5).

- End-to-end realization: We demonstrate an end-toend realization of an IBR architecture: client-side extensions for desktop and mobile platforms, support for legacy consumers and publishers, and additional mechanisms to enhance the intrinsic verifiability offered by IBRs $(\S 6, \S 7)$.

Using a combination of public video and image datasets and end-to-end experiments, we show that IBRs are practical and offer significant benefits ( $(8)$. Specifically, we show that IBRs can offer significant improvements in quality of experience: reducing the video startup delay by $4-9$ seconds, increasing content accessibility, and avoiding rebuffering events (from $50 \%$ of time spent buffering to zero). We also show that using IBRs are practical - web pages rewritten to use IBRs incur low load time overhead $(\approx 0.5 \mathrm{~s})$, IBRs incur close to zero false positives for content naturally occurring in the wild, and IBRs can be resolved efficiently using our system ( $\approx 1$ million lookups/sec on a 25 -node cluster).

\section{MOTIVATION}

We show empirical evidence of quality of experience issues that users face today in accessing shared video links. We also highlight the (unrealized) promise of leveraging alternate versions of the same content from different sites.

Quality issues in shared video links: Recent analysis shows that social media applications are the dominant mode through which users discover mobile videos [7]. We collected the top-500 most popular video links posted on reddit.com and found that $\approx 10 \%$ of the videos are hosted by small providers (i.e., not on YouTube, Vimeo, DailyMotion). ${ }^{1}$ Such smaller hosting sites typically do not offer mul-

\footnotetext{
${ }^{1}$ In an earlier measurement, we found $\approx 30 \%$ of video links were to smaller providers. Moreover, our analysis focuses on the popular content; we suspect that links to smaller providers may be even more prevalent in the "long-tail" of less popular links.
}

\begin{tabular}{l|l|l|l} 
Provider & iPhone & Android & Laptop \\
\hline comedycentral.com & $\begin{array}{l}\text { Doesn't } \\
\text { play }\end{array}$ & $\begin{array}{l}\text { Has play } \\
\text { button, } \\
\text { but no } \\
\text { video }\end{array}$ & OK \\
\hline complex.com & Buffering & $\begin{array}{l}\text { Doesn't } \\
\text { play }\end{array}$ & Buffering \\
\hline vine.co & OK & $\begin{array}{l}\text { Excessive } \\
\text { buffering }\end{array}$ & OK \\
\hline ctvnews.ca & Additional click to mobile site & OK
\end{tabular}

Table 1: Anecdotal evidence of user experience issues on links shared through social media sites through non-popular video hosting sites. Our goal is not to pinpoint specific providers but show problems symptomatic of the entire video+mobile ecosystem. We use a controlled WiFi setting with the same client-side access bandwidth. The reported problems are found during almost every browsing (total of 10 runs) of the given video link on the given device.

\begin{tabular}{l|ccc||ccc||ccc} 
& \multicolumn{7}{|c}{ Startup latency (seconds) } \\
Device & \multicolumn{3}{|c}{ Video1 } & \multicolumn{3}{c||}{ Video2 } & \multicolumn{3}{c}{ Video3 } \\
& YT & V & DM & YT & V & DM & YT & V & DM \\
\hline iPad2 & 4 & 6 & 10 & 2 & 6 & 15 & n/a & 4 & 4 \\
Laptop & 1 & 1 & 2 & 1 & 2 & 4 & 2 & 2 & 2 \\
iPhone4 & 1 & 3 & 5 & 1 & 2 & fail & n/a & 5 & fail \\
Droid & 5 & fail & 4 & 3 & n/a & 2 & n/a & 4 & 3
\end{tabular}

Table 2: Video startup latency across popular video hosting providers; YT, V, and DM stand for YouTube, Vimeo, and DailyMotion respectively. The " $n$ /a' entries show cases where there was no mobile version available. The "fail" entries represent cases where there appeared to be a video but an error message appears after $30 \mathrm{~s}$. We use a controlled WiFi setting with the same client-side access bandwidth. The reported numbers are averaged over 10 runs.

tiple formats or bitrates. Table 1 summarizes anecdotal evidence of user experience issues on a laptop, iPhone, and an Android device for these video links. We see a diverse range of problems: excessive buffering, video does not play, and the user needs to manually navigate to mobile versions. In some cases, we even see problems accessing videos on the laptop, possibly because of server-side issues. Under low bandwidth connections (512 Kbps or less), we see multiple buffering events while viewing these video links as the video content providers typically do not offer lower bitrates for such conditions.

Even popular providers have QoE issues: The quality problems are not just restricted to the lower end of the video ecosystem - even well-provisioned sites such as YouTube, DailyMotion, and Vimeo suffer user QoE and accessibility issues. We found three separate videos hosted on all providers and attempted to access these from four different devices. We focus on the startup delay (i.e., difference between the time when user hits the play button to the time the video starts to play) because it is an important measure of QoE that impacts user retention rates [21, 27]. Table 2 shows the startup delay across the combinations of device, video, and hosting site. First, in many cases the video was not available for mobile devices or failed to play. Second, no 


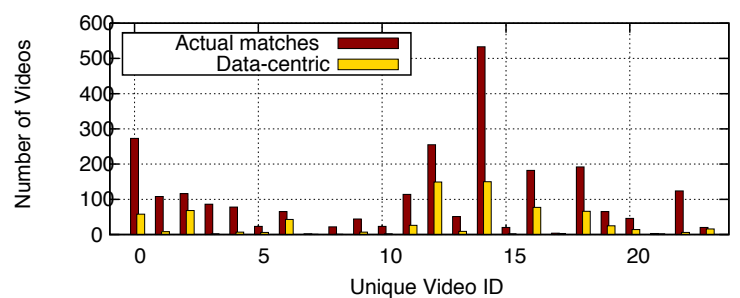

Figure 1: Using a public video dataset, we (manually) verified that are indeed several alternatives for the same video. We also see that data-centric approaches miss many opportunities for leveraging these alternatives as they are tightly bound to the byte-level representations.

specific video hosting site is the best choice across all platforms; e.g., YouTube is good for laptops but not for mobile devices. Third, there is also some diversity across videos w.r.t. accessibility and performance; e.g., Video1 seems to be generally accessible on the iPhone but Video2/Video3 seem to have some issues (no mobile version available or fail to play). We confirmed that these results can be reproduced across multiple network locations and for several other videos (not shown for brevity).

Alternatives exist, but cannot be exploited: While the above results show evidence of QoE problems, they also suggest some hope. For instance, even though Video2 fails with YouTube and DailyMotion on the iPhone, we can potentially stream the content from Vimeo. Measurements have shown that this is indeed the case - there are alternate versions of the same content in different resolutions and encoding formats available on third-party sites [2, 32]. Using a public video dataset [2], we analyze the number of variants of the same video (denoted by a unique video ID) in Figure 1 depicted as "Actual Match". The challenge, however, is that users do not have a way to automatically discover these variants. Even if they do, they may have to manually check that these alternatives meet the intent (e.g., it is not "rickrolling" them) and that these are customized to meet their device and network constraints.

There is a natural parallel here to the motivation for datacentric architectures - users ultimately care about the data and not who is serving it $[36,26,38]$. Thus, data-centric schemes appear to be a seemingly natural strawman solution. Using the same video dataset [2], Figure 1 also shows the inability of data-centric approaches to identify variants of the same video. The reason is that the hashing algorithms underlying data-centric schemes operate at the byte-level. Because minor encoding differences can lead to drastically different byte-level representations, data-centric approaches cannot identify these variants as they only capture exact byte-level copies. ${ }^{2}$

Summary: In summary, we observe that users face significant accessibility and quality of experience issues in accessing video content as the specific links are not suitable for their devices. While there are alternative versions of the desired content on other hosting sites, users cannot reliably discover and leverage these; even future data-centric architectures fail to adequately capture the available alternatives.

What we ideally need is a service that allows users to flexibly leverage third-party alternatives to minimize QoE

\footnotetext{
${ }^{2}$ This is also true for more flexible chunking schemes based
} on Rabin fingerprinting [32].

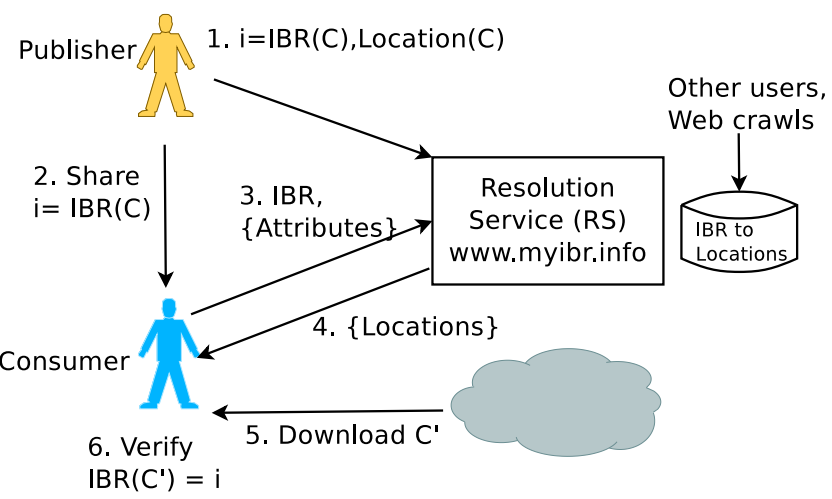

Figure 2: A high-level overview of how IBRs can be used for sharing and retrieving multimedia content.

issues. In fact, many vendors in the mobile and social media ecosystem have immediate incentives and are also naturally positioned to offer such a service. For example, device vendors or service providers can spur adoption without relying on support from content providers. Similarly, social media sites like Reddit or Facebook would also be naturally incentivized to enhance the user experience and thus maximize user retention; e.g., Reddit already runs some (beta) image deduplication services such as karmadecay.com. Alternatively, search engine operators like Google or Bing may also offer such enhanced services specifically targeted to attract mobile traffic; e.g., Google offers the Mobilizer service to create mobile-friendly websites.

\section{ARCHITECTURE OVERVIEW}

Our overarching vision is to enable users to flexibly discover and leverage the alternative versions of the content that can deliver the best delivery performance (e.g., accessibility, bitrate, buffering, startup delay). In this section, we begin with a high-level overview of our approach and highlight the main challenges involved in realizing this vision.

Figure 2 shows an overview of our framework. Today, publishers share multimedia content via URLs that link to hosting sites. As we discussed earlier, this constrains the accessibility and usability of this content by restricting users (e.g., their friends on social networks) to the particular hosting site. Rather than tightly couple references to particular hosting sites, formats, and encodings, we envision publishers who instead post an information-bound reference (IBR) that satisfy two key properties:

(1) Encoding invariant: Different formats and resolutions of the same content map to the same IBR; and

(2) Bound to the information: Unique contents should have different IBRs.

This IBR will be posted via a resolution service $(R S)$; e.g., available at www.myibr.info in Figure 2. The RS maintains a mapping between an IBR and the locations or hosting sites of different versions of content with this IBR. These mappings can be populated using a combination of two techniques: (1) Each publisher generates an IBR for the content she posts or references, and registers an (IBR, location) tuple with the RS; and (2) The RS crawls the Web to identify variants of the same content. For each such alternative, the RS also registers attributes such as the format and bitrate. Note that our framework is general enough to support multiple such resolution services. As we discussed earlier, 
several players in the mobile/social media ecosystem (e.g., Facebook, reddit, Google, Apple) have natural incentives to deploy such a service and also motivate their users to post IBRs (rather than direct URLs) to enhance user retention and attract more "eyeballs".

Consumers query the RS (e.g., http://www.myibr.info? $i b r=" x y z ")$ to find potential alternatives of the multimedia content that have the same IBR $=x y z$ and can choose a version that better matches their constraints; i.e., use a QoEaware selection strategy. They fetch this content from a suitable hosting site, and verify that this matches the $\mathrm{IBR}=\mathrm{xyz}$. Our goal is to provide consumers a high degree of flexibility to view an alternative encoding that is well-suited to their device and current operating constraints. In the simple case, this flexibility is static; e.g., based on what type of device a consumer is using, what the screen size is, how long would this content take to load, and so on. More generally, this flexibility needs to be dynamic; e.g., switching bitrates or streaming servers due to bandwidth changes, or reducing the bitrate when the battery life becomes low.

While this vision builds on the idea of indirection to delay the binding between the content reference and the actual content served, there are two specific challenges that we need to address in order to realize this vision:

1. Building on now familiar arguments for contention-freeness and verifiability, IBRs must be algorithmically generated from the underlying content, as opposed to, say, humaninput labels [29]. To this end, we identify and synthesize algorithms from multimedia fingerprinting (e.g., [19, 34]) in $\S 4$. We also describe how we can augment the verification guarantees in $\S 6$.

2. We need a scalable resolution infrastructure and efficient mechanisms for enabling clients to exploit the flexibility that IBRs offer. Specifically, the multimedia techniques to identify similar content inherently require "fuzzy" matches (i.e., they are not exact string matches) and, thus, we cannot leverage traditional techniques for building scalable key-value stores (e.g., [20]). Thus, we design a scalable resolution service building on locality sensitive hashing in $\S 5$ and describe practical client-side capabilities for QoE-aware delivery in $\S 7$.

\section{GENERATING AND SHARING IBRs}

In this section, we focus on the first high-level challenge: generating and posting references that are algorithmically bound to the underlying information and invariant across encodings, bitrates etc. We focus primarily on video content as it represents a dominant fraction of Internet traffic [3].

Our key insight here is that we can leverage a rich literature of techniques for multimedia fingerprinting to design IBRs $[19,18]$. Multimedia fingerprinting is used in a variety of applications today: duplicate detection [34] and detecting copyright violations [11]. At a high level, this notion of identifying other multimedia content that is "close" to a given object suggests that such fingerprinting algorithms can serve as a useful starting point. However, our IBR vision raises new system-level challenges related to scalability, performance, and verifiability that do not arise in the traditional multimedia applications. In this section, we show how we synthesize these approaches to address these challenges. We begin by describing how to derive IBRs for images which form the basis for our video IBRs.

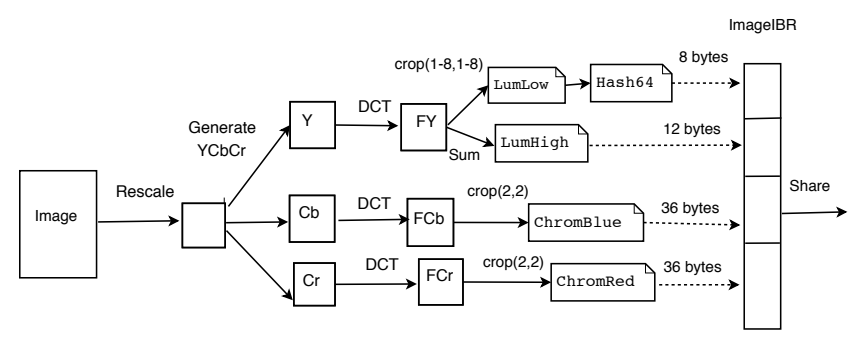

Figure 3: Generating image IBRs. We scale the image to a baseline resolution and convert into the $\mathrm{YCbCr}$ representation [9]. For each component in this representation, we generate summaries to create the IBR.

\subsection{Building Block: Image IBRs}

There are three broad classes of techniques used in image fingerprinting that are based on: (1) understanding spatial structure (e.g., [31]), (2) capturing color distributions (e.g., [30]), and (3) frequency domain analysis (e.g., [19, 6]). The first class of techniques identifies spatial gradients to mimic how the human eye recognizes objects. Color histogram techniques look at the distribution of $R, G, B$ values in an image. These two techniques are coarse-grained and do not capture perceptual differences well. For instance, the spatial techniques do not distinguish grayscale vs. color versions. Similarly, color histograms will be identical for a white-black strip vs. an image with black and white dots scattered uniformly. Thus, we discard these approaches.

The main intuition behind the frequency domain techniques is that the low-frequency components provide a highlevel sketch of the image, and the high-frequency components provide more fine-grained distinctions [15]. Taken together, the resulting fingerprint more closely reflects the underlying information content, making these techniques a good starting point for our system. We found that the existing scheme, pHash [6], was not robust enough to satisfy the requirements of IBRs, so we adapted it as described below.

Figure 3 shows how we generate the IBR for an image using frequency domain techniques. We first scale the image to a baseline resolution of $128 \times 128$. $(128 \times 128$ is a good baseline as it is lower than common image resolutions, but high enough to discern detailed structures.) Then, we generate the YCbCr representation of this scaled image [9]. We run the discrete cosine transform (DCT) on the $\mathrm{Y}, \mathrm{Cb}$, and Cr matrices to get DCT coefficients.

The IBR consists of two parts: (1) We take the lower end submatrix (rows 1-8, columns 1-8) of Y DCT matrix - which capture more than $95 \%$ of the signal energy - and generate a compact 64-bit summary, Hash64, by first finding the median of the coefficients and then quantizing each coefficient to be 0 or 1 depending on whether it is higher or lower than the median [19]. This compact summary reduces the cost of checking if two IBRs are identical. The pHash scheme also uses Hash64. (2) To capture more fine-grained differences, we compute the sum of the high-frequency components of $\mathrm{Y}$, and capture the lower-end $3 \times 3$ sub-matrices of $\mathrm{Cb}$ and Cr DCT components where most of the signal energy lies. The image IBR is the 92 byte concatenation of the Hash64 and other components. The pHash scheme does not capture these fine-grained differences. 


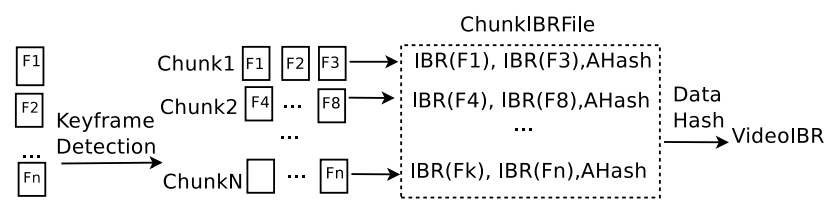

Figure 4: Generating video IBRs. We chunk the video via keyframe detection and compute a per-chunk IBR using the image IBRs of the first/last frames. We apply a traditional data hash to the file containing all the chunk IBRs, and use that hash as the video IBR.

\subsection{Video IBRs}

Next, we discuss how to extend the image IBR to video (Figure 4). One extreme solution is to just think of a video as a sequence of images and simply concatenate the IBR for each frame. However, this makes a video IBR large and expensive to compute. On the other hand, we can use only the image IBRs for the first and last frames. Unfortunately, this allows arbitrary content to be injected between these frames. To ensure tighter binding, it is clear that video IBRs need to encode information about more frames. To achieve this, an intuitive option is that we could sample frames on time/byte boundaries; e.g., every 5th second or every $\mathrm{N}$-th frame. However, sampling-based alternatives are not robust as they are sensitive to variations in timing and encoding formats and can result in inconsistent chunking across variants.

To ensure tighter binding and consistent chunking, we leverage techniques for scene detection to derive the appropriate chunk boundaries from the information itself [18]. These scene detection schemes identify keyframes when the content changes significantly across frames. Intuitively, this is analogous to value sampling in data chunking [25, 32]. To identify the scene changes, we need to select an image feature that is easy to compute and consistent across minor variants. We applied various transformations (e.g., format and bitrate changes) to a sample set of 50 movie trailers from Youtube and observed that using the variation in the amplitude of the zero-th frequency of the $\mathrm{Y}$ component yields consistent boundaries across these video transforms. Thus, our chunking algorithm works as follows: Given the zero-th frequency component $A_{i}$ for each frame $i$, we compute the distance between two successive frames $i$ and $i+1, \operatorname{Dist}(i, i+1)=\frac{\left|A_{i+1}-A_{i}\right|}{\min \left(A_{i+1}, A_{i}\right)}$, and check if this crosses a threshold ChunkThresh.

Each chunk is described by a chunk $I B R$ consisting of two components. The first component is a two-tuple capturing the image IBRs of the start and end frames $\left\langle I_{\text {start }}, I_{\text {end }}\right\rangle$. The second component is a 424 -byte audio IBR that we generate using an existing audio fingerprinting algorithm [5].

A practical issue here is chunk size. Smaller chunks enable more fine-grained adaptation to device and network constraints (e.g., switching to low resolution when battery is low) and provide tighter verifiability (in the limit every frame is a chunk), but also imply more lookup overhead. As a tradeoff between these factors, we set ChunkThresh $=0.5$ based on controlled experiments (not shown), which yields an average chunk size of $\approx 5$ seconds. We also impose a minimum chunk size of 0.5 seconds so that the resolution overhead is low compared to the data transfer time.

Sharing video IBRs: One concern is that an IBR for a long video with many chunks may be too large. For each chunk, the IBR is $\approx 0.6 \mathrm{~KB}$ as each image IBR is 92 bytes and the audio IBR is 424 bytes. Thus, for a 20minute video clip using a 5-second chunk size, the video IBR is roughly $150 \mathrm{~KB}$. Thus, downloading the list of chunk IBRs may increase the video startup delay. We design a practical workaround for this. The IBR that a user posts for a video is analogous to a "torrent" file containing the list of chunk IBRs. (In fact, HTTP chunking based techniques used in video players already do this.) That is, in the IBR www.myibr.info?ibr=xyz for the video "xyz" is a data-centric hash of a manifest file containing the list of chunk IBRs. Note, however, that the data hash is used only to get the per-chunk IBRs. All subsequent actionsresolution/matching, download, and verification-use the per-chunk IBRs which by design bind to the information.

Using this manifest file containing per-chunk IBRs has two immediate advantages. First, it allows players (or client plugins) to download each chunk IBR in parallel while streaming the video, effectively hiding the latency of downloading the IBRs. Second, it also allows dynamic adaptation on a chunk-level granularity similar to HTTP chunking and DASH [12].

There are other practical benefits of using chunk-level IBR resolution. It can help to find more variants of the video chunks, since full video IBR need not match. In addition, it can accommodate content insertions (e.g., advertisements) in the video. Because of our keyframe detection, ads would be considered as different chunks, and we could still find variants for the chunks of the actual content.

\section{IBR RESOLUTION}

Having generated IBRs, the next step is to match IBRs in order to resolve the references to the final content that the consumer will view. We begin by discussing the algorithm for matching two IBRs. Then, we describe the design of a scalable resolution service for matching IBRs. We also discuss how we support legacy users and publishers.

\subsection{Matching IBRs}

Matching video chunk IBRs largely boils down to matching the constituent image IBRs. Thus, we begin by describing how to match two image IBRs. The Hash64 (Figure 3) provides a quick check to distinguish two IBRs. However, Hash64 values may differ slightly across different lossy encodings of the same image. To reduce the likelihood of false negatives (i.e., the $\mathrm{RS}$ is unable to find alternatives even though they exist), the matching process has to accommodate some fuzziness. To this end, we compute the Hamming distance between the two 64-bit values and check if it is within a threshold.

If the Hash64 fields match, we proceed to match the remaining image IBR attributes. Again, these matches are fuzzy; if the differences are smaller than specific thresholds, we classify the images as identical. The use of thresholds in the matching process naturally implies choosing them carefully to control false positives and false negatives. We explain how we tune these thresholds and how they perform on real-world datasets in $\S 8$. 


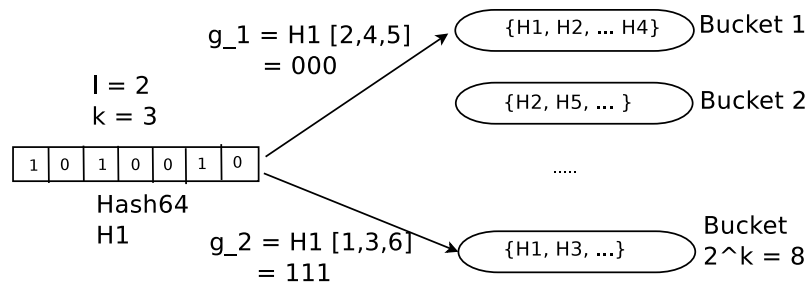

Figure 5: Bit sampling for LSH with $l=2$ hash functions and $k=3$ bits per hash. Here, the hash function $g_{1}$ chooses bits at positions 2,4 and 5, while $g_{2}$ picks bits at positions 1, 3, and 6 .

\subsection{Locality-Sensitive Hashing}

Given the popularity of video content, we expect RSes to process IBR resolution requests at a high rate. The key issue that makes this challenging, as discussed above, is that IBRs generated across different encodings might differ slightly. This is not an artifact of our generation algorithms, but inherent to video content and the lossy encodings and transforms applied to them. Thus, the RS must support fuzzy matches. A naive RS design can impose high resolution latencies that impact user experience. Our initial experiences with a MySQL-based backend implementation with user-defined functions could only support $\approx 150$ queries per second even on a high-end server.

Our first step toward scalable fuzzy matching is to use Locality Sensitive Hashing (LSH) [13]. The high-level idea in $\mathrm{LSH}$ is to treat the fingerprints as high-dimensional distance vectors and project them to a smaller dimension. To find the nearest neighbors, LSH only compares vectors in this low-dimensional projection, which is significantly cheaper than comparing them in their original representation. The intuition is that similar vectors will (with high probability) be similar in the low-dimensional projection.

Our IBR matching problem is similar at a high-levelwe want to find IBRs that are close to a given query. Thus, LSH is a promising starting point. In our setting, we want to compare the Hamming distance between the Hash64 fields. In this case, an efficient way to implement LSH is via bit sampling [13], which when applied to matching the Hash64 fields works as follows (Figure 5). We define $l$ hash functions $g_{i}, i=1 \ldots l$, where each $g_{i}$ takes in as input a Hash64 $H$, selects $k$ random positions from $H$, and outputs a $\mathrm{k}$-bit vector which is the concatenation of the values at these locations. Then, we map $H$ to the logical buckets corresponding to the values of $g_{1}(H), \ldots g_{l}(H)$. When a query for $H^{\prime}$ arrives, we retrieve the entries in the buckets $g_{1}\left(H^{\prime}\right), \ldots, g_{l}\left(H^{\prime}\right)$. Then, we compute the exact Hamming distance between $H^{\prime}$ and each entry in these buckets to identify potential matches. The intuition is that if $H$ and $H^{\prime}$ are close enough in terms of Hamming distance, they are likely to match when we consider their bits in some $k$ random positions. By choosing $l$ such hash functions, we increase the likelihood of finding such matches.

Extending to video chunk IBRs, we obtain candidate chunk IBRs using an LSH-based check for the first frame of the queried chunk IBR, and directly compare the other IBR fields of these candidates with the input IBR.

There is a tradeoff between two key metrics: the number of entries that need to be processed per input query (and thus the overall throughput) and the false negative rate (i.e., there are candidate IBRs close to a queried IBR, but none of their $l$ hash values match). This tradeoff depends on the choice of $l$ (the number of hash functions) and $k$ (the number of bits per hash function) [13]. Small $l$ and large $k$ can increase throughput, but increase false negative rate. On the other hand, large $l$ and small $k$ can decrease false rate, but at the expense of decreasing throughput. In practice, using a real world video dataset [2], we find that $l=20$ and $k=20$ is a reasonable point in the space of tradeoffs. With this setting, we can find close to $90 \%$ of the alternatives at a rate of throughput of $8 \mathrm{~K}$ queries/second. Note that our overall goal here is to find some candidate set of suitable alternatives that meets the users' constraints; we do not need perfect recall.

\subsection{Performance Improvements}

Next, we explore opportunities to improve the throughput, reduce false negative rates and scale the RS.

Heuristics: We use three heuristic improvements to improve throughput and reduce false negatives.

- Pruning: The first optimization is to stop the search after a sufficient number of matches meeting the consumerspecified constraints are found. This reduces the number of comparisons, thereby improving latency. To avoid the same matches for all queries and to be "fair" to different hosting sites, we randomize the order of hashtable lookups.

- Pre-clustering: We group nearby IBRs into clusters in an offline pre-processing stage. This helps reduce false negatives during lookup. When a matching IBR is found, we retrieve the pre-computed cluster and directly compare all the IBRs in this cluster. Thus, we identify all potential IBR matches (no false negatives), if at least one of the IBRs in a cluster has one of the $l$ hash values matching the query IBR. This optimization also improves throughput because we stop the search after finding one such cluster, avoiding further lookups. If the query IBR is already present ${ }^{3}$ in the RS, this optimization results in only one lookup.

- Bypassing LSH: The last optimization exploits typical viewing patterns where users typically watch a video in sequence. Further, in the common case we expect videos to be whole matches of each other; i.e., if a chunk IBR in a video matches the chunk IBR in another video, it is likely that the subsequent chunk IBRs of the two videos would also match. We exploit this structure to bypass the LSH step. Here, for each chunk IBR, we maintain metadata about its parent manifest file (which contains the list of chunk IBRs) and its offset there. As before, we use a LSH-based match for the first chunk. Having identified candidate IBRs for the first chunk, we retrieve their corresponding manifest files. For a subsequent lookup to the chunk at offset $j$, we fetch the chunks at offset $j$ from these matching chunk-list files, and directly match these IBRs bypassing the LSH stage. If these don't yield sufficient matches (say, because the video is a mashup of scenes from different videos), we fallback to LSH search.

$\S 8.3$ provides a breakdown and analysis of the scalability improvements from each of these optimizations using real-

\footnotetext{
${ }^{3}$ In general, a query IBR may not be present. For example, an IBR is registered to one RS by a content publisher, but it is queried against another RS maintained by a device vendor
} 
world datasets. With these in place, the throughput improves to $30-45 \mathrm{~K}$ queries per second on a single server.

Parallelization: To scale the RS further, we use a simple parallelization strategy. We partition the address space of LSH hashtables (i.e., the $g_{i}$ logical buckets ) across different machines. Thus, different hashtable lookups would be assigned to different machines. As discussed earlier, each query involves lookups from up to $l$ hashtables; we simply randomize the (permutation) order in which we lookup the hashtables for each query. For example, query1 may proceed in the sequence $1-2-5-\ldots$, query2 may proceed in sequence 3-1-9-..., and so on. (This is implemented by a lightweight front-end load balancer.) This ensures that the queries are executed in parallel across machines as much as possible.

To put this in context, YouTube serves $\approx 2$ billion videos per day [10]. Assuming an average video length of 5 min and 5 seconds/chunk, this translates to approximately 1 million chunk requests per second. Given that we achieve $45 \mathrm{~K}$ queries/sec on a single machine, a cluster of 25 machines can support this YouTube-scale workload of 1 million queries/sec.

\subsection{Legacy users and publishers}

To support legacy publishers, the RS maintains inverse mappings between URLs and IBRs. It supports an additional query interface, where user devices provide direct URLs instead of IBRs. For such URL queries, the RS performs an extra lookup to first find the IBR (a simple exact match) and then identifies variants using this IBR.

Legacy users who want to access content from IBR-enabled publishers direct their requests via an IBR-enabled proxy. If they need simpler static customization, the RS runs some implicit content negotiation (e.g., using UserAgent strings) and also allows them to set preferences regarding bandwidth and resolution which are sent as cookie values with subsequent HTTP requests. The RS issues a traditional HTTP redirect to a suitable alternative. In order to access content from legacy publishers, however, the requests from legacy users need to go via our proxy. (Otherwise, it will fetch the URL directly.)

In short, while our framework supports legacy users, we get maximum benefits when users and publishers are IBR enabled. Legacy users also need to trust additional entities (e.g., proxy, RS) to achieve the same degree of verifiability.

\section{VERIFIABILITY}

Being bound to the underlying information helps IBRs provide intrinsic verifiability. We discuss the verification step and mechanisms to improve the verifiability further.

Client-side verification: As a video chunk is downloaded, we generate its IBR and match it against the intended chunk IBR. This occurs in parallel as the client is downloading future video chunks. The verification step provides resilence against attacks where an attacker tries to claim that some bogus content matches a given IBR. The actual overhead of verification is quite low beyond the cost of decoding the content which the device will incur anyway.

One potential concern is that an attacker can exhaust the client's resources to download, decode, and verify fake content; i.e., the IBR claims to be video $\mathrm{X}$ but the actual IBRs of the chunks within the video do not match the IBRs. While this is a valid concern, we note that this attack's power is bounded. As soon as the client detects a bogus video chunk from a URL, it can stop downloading chunks from that URL and uses alternate sources for the remaining chunk IBRs. Thus, the wasted bandwidth is only for the bogus chunk; i.e., approximately 5 seconds worth of bytes. As a further protection against such bandwidth-exhaustion attacks, users can report verification failures to the RS. The RS can revoke these fake mapping, after further checks where it locally computes the IBRs, to avoid using these mappings for future requests.

There are still two possible weaknesses: 1) a determined attacker registers malformed content having the same IBR as some genuine content, and 2) unintentional IBR collisions between content from genuine providers. Next, we discuss mechanisms to overcome these issues.

Access control via scopes: When the publisher registers her IBR and content at the resolution service, she also annotates it with a logical scope. These scope annotations allow publishers to constrain the set of third-party providers who can be candidates for serving the content. For example, the publisher may only allow trusted third party providers for high-priority content. (We assume that each provider can be identified; e.g., via a email-id or an identifier in the social network.) For low priority content, it may register a scope with a wildcard to allow anyone to register. When a thirdparty provider tries to register an IBR-to-URL mapping, the $\mathrm{RS}$ checks whether this provider belongs in the given scope, and can accordingly allow or drop this mapping. If the publisher detects misbehavior from some specific provider, she can blacklist this third party and ask RS to remove it from the scope.

Larger or multiple IBRs: In general, using larger IBRs provides tighter binding to the information. For video IBRs, we can add additional components to the per-chunk IBR that capture the variation across frames within a chunk to protect against frame addition or deletion attacks. Instead of choosing a fixed size IBR, publishers can provide two IBRs: (1) a compact IBR used for publishing the references on webpages, and tweets and (2) a larger IBR made available out-of-band (e.g., along with the chunk list file for the video). Consumers can use the larger IBRs for extra verification.

We also envision using multiple complementary IBR algorithms to improve verifiability as different fingerprints capture different characteristics (e.g., spatial gradients vs. colors). In this case, the publisher provides multiple IBRs with annotations to identify the specific algorithm in use, and the consumers verify the IBR for each version. The likelihood of IBR collisions on all versions should be low and thus improve the verifiability guarantees.

\section{IMPLEMENTATION}

IBR Generation: We implement the generation algorithms by extending the pHash library [6] involving roughly $2.5 \mathrm{~K}$ lines of $\mathrm{C}++$ code. We leverage off-the-shelf audio fingerprinting algorithms [5].

Resolution Service: We built a functional resolution service using a PHP-based web frontend running on top of Apache integrated with our optimized LSH-based backend. We use a C++ based LSH backend consisting of roughly 600 lines of code. Our current prototype optimizes three metrics to improve user experience: load time for videos, buffering, 
and number of "user clicks" required to play the video on the device. Our goal in this paper is not to devise an optimal policy to balance these metrics; rather we provide a mechanism for flexible adaptation. We support both mechanisms outlined in $\S 3$ to populate the RS; the RS verifies the binding between content hosted at the URLs and the IBRs before adding IBR-to-URL mappings.

Client-side extension for QoE-aware delivery: One possibility for enabling content negotiation between consumers and the RS is using conventional HTTP Accept: headers. However, this option is not expressive enough; the existing standard only allows the ability to specify preferences for certain encodings, e.g., Accept: video/mpg. Finer grained controls, e.g, a $3 \mathrm{G}$ user wanting bitrate $\leq 200 \mathrm{kbps}$, are not possible.

To provide such fine-grained controls, we need client-side modifications. To this end, we developed a Firefox browser extension using Greasemonkey [4], a popular page rewriting tool. The extension processes IBR-ized links on the publisher's site and contacts the RS. It analyzes the available media codecs and plugins, and local conditions such as device type, the type of network interface in use, available bandwidth and battery state, and provides this information to the RS. It rewrites the HTML depending on the RS's response. When the browser loads the new HTML, it simply issues GETs to appropriate URLs. For pages with several video references, we batch requests to the RS to avoid multiple round trip delays. Our prototype currently supports three attributes for content negotiation: device type, format, and bitrate.

The plugin masks the latency of downloading the perchunk IBRs by fetching them in parallel along with the video stream. When the $\mathrm{RS}$ reports multiple alternatives for a chunk, the plugin prefers chunks on the same hosting server and in the same format, unless forced to switch servers because of consistently poor performance. Similarly, when network or device conditions necessitate a change in bitrates, the plugin avoids drastic shifts, choosing instead a feasible bitrate closest to the previously viewed bitrate. The plugin also provides (anonymous) feedback to the RS to track QoE issues specific to a video or hosting site on that device.

Android implementation: We also implemented clientside capabilities for Android (v4.0, Ice Cream Sandwich). In order to provide the IBR functionality in an app-independent fashion, we modify the MediaPlayer module, which is part of the webkit middleware. We add the IBR logic to the setDataSource function in this module that selects the URL to play. We interpose on this call, and receive an updated URL from the IBR resolver. We use standard APIs to obtain current operating conditions (e.g., Connectivity Manager to identify WiFi vs. 3G, Window- ManagerDisplay to get resolution, and BatteryManager to get the battery status) and report them to the resolver. One concern is that modifying webkit likely requires a device/OS upgrade; we envision this is a feasible option for device vendors and wireless providers who already customize devices.

Proxy: To support legacy clients (§5.4), we also implemented a simple proxy in $\mathrm{C}++$ that essentially replicates the functions that the client-side browser extension performs while communicating with the RS.

\section{EVALUATION}

We address the following questions in our evaluation:

(i) QoE Improvements: Can IBRs enhance user experience (e.g., load time, buffering)? (§8.1)

(ii) Generation: How do we configure IBRs to ensure low false positives and false negatives? How effective are IBRs in identifying similar/dissimilar content in the wild? (§8.2)

(iii) Resolution: Can the RS resolve IBRs quickly and for a large number of users simultaneously? ( $\$ 8.3)$

(iv) Overhead: Do users perceive delays in viewing pages authored with IBRs? ( $\$ 8.4)$

(v) Verifiability: How fast can users check IBRs? How practical are the verification guarantees offered by IBRs? (§8.5)

\subsection{QoE improvements using IBRs}

We begin by showing the quantitative benefits that IBRs provide consumers in realistic settings on actual devices. In particular, we show how IBRs can enable network- and device-specific adaptation to improve the user experience.

Bandwidth adaptation: We emulate a blog with an embedded video object in mpg format with a bitrate of 1.2 Mbps. We assume that there is an alternative encoding at $620 \mathrm{Kbps}$, from alternate source. We use a browser with a VLC plugin to play the video and a WAN emulator to vary the download speed between 512Kbps to $2 \mathrm{Mbps}$.

As a baseline, we consider today's publisher/consumer setup with no IBR support. Here, the publisher uses a video URL that supports single format and resolution ( $(2)$. At low bandwidth $(768 \mathrm{Kbps})$, we observed $6-10$ pauses while viewing the video and significant buffering (only 15 s played over a 30 s period). Next, we test the case when the publisher chooses to share the video using IBRs and the client uses our updated browser extension. The video was played smoothly without any buffering induced pauses in this case. At high bandwidth (1.5 Mbps), the browser extension detects and sends this bandwidth information to the RS, which redirects it to the high quality variant. This preliminary result under a controlled setting suggests that IBR-enabled clients can have a better user experience by adapting to dynamic network conditions.

Device adaptation: We emulate an experiment with users sharing video links on social networking sites and consumers viewing those posted links on different devices. For this experiment, we use the same example videos from Table 2. We post them on Facebook and use IBRs to share them. In the case of mobile devices without our browser extension, the RS uses implicit content negotiation to redirect clients to the hosting site best suited for the device.

As before, we quantify the end-to-end user experience on the devices w.r.t startup latency for the video to play. Because IBRs redirect users to videos which can play on these devices, it significantly improves content availability; e.g., the third video URL from YouTube does not play on any of the mobile devices, but using IBRs the content can be retrieved from one of the other locations. Furthermore, using IBRs reduces the join time by up to 1 second for Droid on YouTube, up to 4 seconds for Vimeo on iPad2 and up to 19 seconds for DailyMotion on iPhone4 (not shown).

These experiments suggest that using IBRs can improve the viewing quality of experience by reducing join time, buffering, and avoiding scenarios where videos were not viewable. 


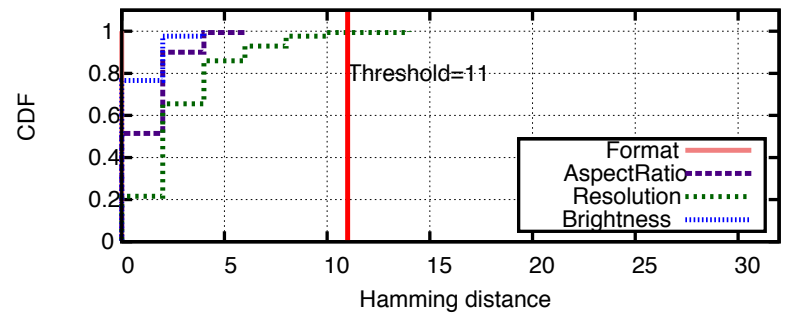

(a) Across transforms

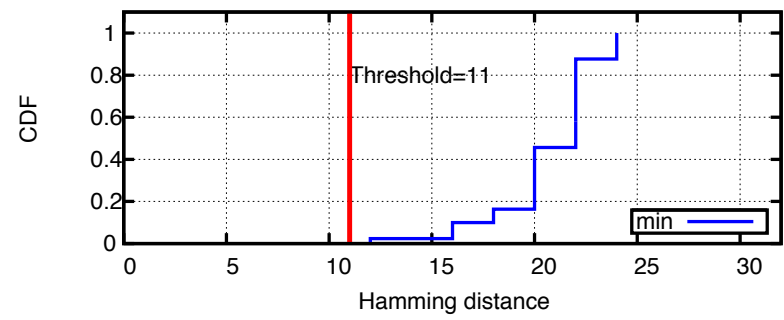

(b) Across distinct images

Figure 6: Threshold for Hamming distance. This plot compares the Hamming distances of the Hash64 field across transformed versions of images and distinct images, and shows that we can set a threshold of 11 .

\subsection{Configuring IBRs}

Next, we study how to control the degree of false positives and false negatives introduced when matching different video IBRs. Recall from $\S 4.2$ that a video IBR is essentially a sequence of chunk IBRs, where each chunk IBR has image IBRs of the start and end frames. Thus, we begin by focusing on configuring image IBRs before proceeding to video-specific configurations.

Configuring thresholds for image IBRs: Ideally, we want IBRs that yield zero false positives (i.e., not mark distinct images as same) and zero false negatives (i.e., not mark two identical images as different). We show that it is possible to choose IBR matching thresholds to get close to this ideal. In this process, we are willing to tolerate a small increase in false negatives in favor of completely avoiding false positives. In other words, we are trading off a small decrease in availability for guaranteeing correctness.

As our training set, we used the Univ. Washington image dataset consisting of real-world scenes of nature, people, events, and plants [8]. For each image, we apply the following transforms: (1) change format (from JPEG to BMP and PNG), (2) change resolution (scaled to one-third), (3) change aspect ratio (converting to 1:1), and (4) increase brightness. We chose these specific transforms because we observe that these occur commonly in the wild [2].

Figure 6(a) shows that the Hamming distance between Hash64 components across transformed variants is at most 15. Figure 6(b) shows that the minimum Hamming distance across different images is at least 13; i.e., for all images, the "nearest" distinct image is at least at a distance of 13. Based on this, we set a threshold of 11 on the Hamming distance between Hash64 to minimize the false negative rate while maintaining a zero false positive rate. In similar fashion, we use this dataset to select thresholds for other finer grained

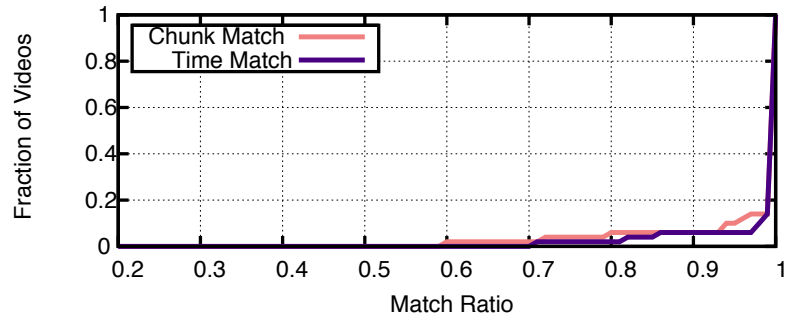

Figure 7: Controlled dataset study for videos. This shows the distribution of match ratio (time/\# chunks) across two video formats. The results are similar for variants that differ in the resolution.

components of the IBR (details omitted for brevity). With these thresholds, we get a zero false positive rate and a false negative rate of $0.4 \%$. We validated these thresholds on a different set of 250 images from the same dataset and 4223 images from a different dataset [1], and found $1.3 \%$ and zero false negative rates respectively and no false positives.

Video IBRs: Having chosen the image IBR thresholds, we move to video IBRs. First, we use a controlled dataset of 50 movie trailers from Youtube to analyze the effect of chunking on the match rate across transformed variants of a video. We apply two transforms: changing the format from $f l v$ to avi and rescaling to $200 \times 180$. Figure 7 shows the distribution of the match ratios across videos with the format change. The result shows match ratio w.r.t. time (ratio of total time of matched chunks and total video time length) and number of chunks. We see that format changes have minimal impact; the match rate is $\geq 95 \%$ for more than $95 \%$ of the videos, both w.r.t. time and chunks. Most match misses occur from a known corner case with blank screens where the Hamming distances between similar frames becomes high; we handle this corner case separately, by forcing the chunking algorithm to choose non-blank frames for the first/last chunks, to further reduce false negatives. The result for the resolution change are similar; we do not show this for brevity. To understand how chunking affects match rate, we disable chunking and do a per-frame match and find that the difference between chunk- and frame-level match rates is $<0.5 \%$ (not shown). This shows that our chunking algorithm yields consistent chunks across video transforms.

Video IBRs in the wild: Moving beyond controlled tests, we run the video IBR algorithms on two larger multimedia datasets collected in the wild: (1) 13,123 videos fetched using 24 popular queries for a "seed video" (CC Web Video dataset) [2]. ${ }^{4}$ (2) 120 videos downloaded by querying for a popular movie title across torrent sites (SET dataset) that we manually labeled [32].

We identify two videos as similar only if all their chunk IBRs match. Then, we verify this against manually labeled ground truth. Figure 8 summarizes this result for the CC Web video dataset; "Text-based" represents the total number of results obtained by the query and "Actual" represents the number of videos that were manually labeled as similar. The difference between "Actual" and "Text-based" indicates the noisy results from text based queries. IBRs did not identify any of the noisy results as similar (i.e., zero false

${ }^{4}$ This is the largest manually labeled video dataset we are aware of. 


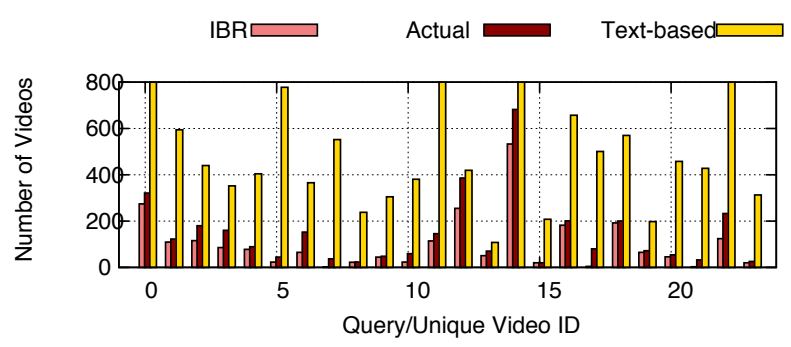

Figure 8: IBR effectiveness in the wild. This compares matches found by IBR with the actual matches on datasets collected in the wild. It also shows the noisy results from text-based queries.

positives). Using the IBRs as-is identifies $72 \%$ of the similar videos. Again, the misses were due to the previously mentioned corner case of blank screens. Using the above heuristic to handle this case reduces the false negative rate to less than $5 \%$. Our results for the SET dataset are similar (not shown): zero false positives and roughly $5 \%$ false negatives. These results show that IBRs are effective and robust "in the wild".

\subsection{Resolution Performance}

Scalability: We measure the query lookup performance of our LSH-based RS (with and without the optimizations in §5) using a single core on a Intel(R) Core2 Quad Q6700 2.6 $\mathrm{GHz}$ CPU. For this experiment, we use the IBRs for 80,000 chunks from the CC Web Video dataset to populate the RS. For each chunk, we insert half the available alternatives into the RS, and use the other half as query inputs. The entire LSH data structure resides in main memory. We used $l=20$ hash tables and $k=20$ bits per hash function.

Using the basic LSH structure without any optimizations, we obtain $8 \mathrm{~K}$ chunk queries/sec. As discussed in $\$ 5$, even this is several orders of magnitude faster than a traditional database backend. Pruning the number of query results to stop after 50 matches are found, improves the throughput to $18.5 \mathrm{~K}$ queries/sec. Pre-clustering (without pruning) reduces hash table lookups as the LSH search can be stopped as soon as a cluster is found. In particular, we find that the number of hash table lookups reduces to 9 on average across queries compared to 20 for the basic LSH where all hash tables need to be looked up. For requests that had at least one match, we need only 1.6 lookups on average. Thus, pre-clustering improves throughput to $20 \mathrm{~K}$ queries/sec. After combining pre-clustering with the above pruning threshold of 50 , the throughput increased to $30 \mathrm{~K} / \mathrm{sec}$.

We noted in $\S 5$ that we could bypass the LSH step for lookups to subsequent chunks within the same video. The SET dataset had larger clips (15 minutes on average) and several of these were chunked by our algorithm resulting in roughly 80,000 chunks across all video clips. As before, we divide these equally into chunks stored in the mapping and queries. The baseline LSH throughput was $8 \mathrm{~K} / \mathrm{s}$ that improves to $20 \mathrm{~K} / \mathrm{s}$ with preclustering. Bypassing the LSH for subsequent (sequential) lookups within the same video, improved the throughput to $35 \mathrm{~K}$ queries/sec. When we combined pre-clustering on the first few chunks together with

\begin{tabular}{l|c|c|c} 
Template & \# Objects & Load time (s) & Increase with IBR (s) \\
\hline T1 & 1 & 0.2 & 0.1 \\
T2 & 25 & 0.6 & 0.3 \\
T3 & 50 & 0.8 & 0.5
\end{tabular}

Table 3: Overhead of using IBR-ized websites compared to regular URL versions for three website templates.

this bypass optimization, the performance improved further to $45 \mathrm{~K}$ queries $/$ sec. $^{5}$

False negatives: As discussed in $\S 5$, the LSH approach could result in false negatives. Using the basic LSH framework on the CC Web Video dataset, we saw a miss rate of $14 \%$. These misses fall in two classes: (1) a queried IBR matches some variants but not others and (2) the query yields no matches although similar variants exist. Fortunately, $96 \%$ of all misses are of type (1), and can be addressed via the pre-clustering optimization. We can further address misses of type (2) using one of two extensions: smaller $k$ to reduce the likelihood of misses and using multiple parallel LSH data structures with different random seeds.

\subsection{Increase in page load times}

Next, we evaluate the overhead that a user may experience in viewing pages (e.g., blogs) with IBR-ized links due to the need for multiple IBR resolutions. We created three template web pages from blogging and social networking sites, varying in the number of links to multimedia objects. We assume that there is only one version of each embedded object and all objects are hosted at a single remote server. To be conservative in estimating the overhead, all requests go through the RS even though there are no IBR-induced benefits from adaptation. We used a WAN emulator to simulate an average latency of $60 \mathrm{~ms}$ between client and the RS.

As Table 3 shows, while the IBR-ized version does marginally increase the page load times (mostly because of the additional RTT to contact the RS), the worst case load time is an order of magnitude lower than suggested user tolerance (2 seconds) [22]. This overhead can be reduced even further by optimizing the PHP-based RS or using other web page optimization techniques. Finally, it is important to note that this small overhead will be offset by the performance benefits we saw in $\S 8.1$.

\subsection{Verifiability}

The previous sections showed the effectiveness and usability of IBRs in normal operating conditions. The final issue we consider is how consumers can verify if the content they view matches the publisher's intent and if they can detect targeted content pollution attacks.

Overhead: The first concern is verification performance. Here, the design of video IBRs facilitates rapid checking: On an Intel Core2 2.66Ghz machine, it takes $0.02 \mathrm{~s}$ to extract the start/end frames of a chunk, $0.2 \mathrm{~s}$ to compute the image IBR for each, and $0.01 \mathrm{~s}$ to compare against the original chunk IBR. Thus, the total time for verification is $0.25 \mathrm{~s}$ per-chunk; this latency can be masked by running these checks in parallel with downloading future chunks. On mobile devices, we expect that this step can be done in hardware as most

\footnotetext{
${ }^{5}$ The $\mathrm{CC}$ Web video dataset had small videos ( $<5$ chunks); thus we do not report numbers for this experiment.
} 


\begin{tabular}{l|l|l} 
Attack & Description & Verifiability? \\
\hline Inset & $\begin{array}{l}\text { embed bogus con- } \\
\text { tent }\end{array}$ & LumLow \\
Quantization & $\begin{array}{l}\text { poor qual- } \\
\text { ity/large pixels }\end{array}$ & ChromBlue,ChromRed \\
Resize & $\begin{array}{l}\text { rescale image, } \\
\text { then magnify }\end{array}$ & LumHigh \\
Small text insert & $\begin{array}{l}\text { random text } \\
\text { replace small } \\
\text { Replace faces }\end{array}$ & none \\
& none
\end{tabular}

Table 4: Attacks against the image IBR

smartphones and tablets already use hardware-assisted decoding for video.

Resilience to pollution: IBRs can easily protect against different content; e.g., against "rickroll"-style attacks in social media. While this eliminates obvious violations, IBRs could be prone to subtle pollution attacks. Next, we study the robustness of our IBRs against such attacks.

Table 4 summarizes different emulated "attacks". Note that these are subtle attacks; attacks that modify the content substantially will be detected as the Hash64 would differ. We see that some of these attacks are detected via the fine-grained components of the IBR such as the ChromBlue, ChromRed, or the LumLow values (Figure 3 in $\S 4$ ) even if Hash64 fails.

In addition to approaches outlined in $\S 6$, one approach to tackle such subtle pollution attempts is via a humanassisted reputation service [40]. To analyze the viability of such a service, we recruited 100 users and showed them a series of $n$ two images. Users vote if these images are identical or different. We pick a random frame from the original and potentially polluted videos. To avoid biasing users, we advertised this as a generic study on "image perception" and also randomly interspersed identical pairs of frames along with modified pairs. We focus on the text/face scenarios from Table 4 and found that the observed probability of detecting these attacks is $\geq 0.8$ (not shown). Furthermore, users rarely voted identical frames as different. This provides preliminary but promising evidence to complement the verifiability offered by IBRs.

Video IBRs naturally inherit the verifiability properties of image IBRs. An additional concern with videos are frame insertion (e.g., bogus frames for ads), frame replay, and frame permutation attacks. To address these, we use larger chunk IBRs and add a Hash64 for every frame in the chunk. To compare chunk IBRs, we compute the pairwise Hamming distances between pairs of corresponding frames, and check if the maximum pairwise distance exceeds a threshold. Under all intra-chunk attacks, this value was $\geq 21$ confirming the additional robustness offered by larger IBRs.

\section{RELATED WORK}

Use of multimedia techniques in networking: The original vision of IBRs was outlined in an earlier position paper [16]. This work presents a more careful synthesis of the multimedia algorithms, a system for scalable IBR resolution, and an end-to-end implementation. Other recent work has leveraged multimedia algorithms in the context of disaster-recovery applications [41, 37]. These efforts focus on images and largely use the algorithms as deduplication tools. Furthermore, these do not address issues w.r.t. resolution and do not present a full system realization.
Content-centric networking: Recent work argues that we do not need significant network upgrades to achieve the security and performance benefits of data-centric schemes [23]. Our vision is not in conflict with these arguments. First, we do not make a case for ubiquitous caching; rather we exploit alternatives at third-party sites that exist already. Second, the benefits of IBRs can be achieved in a backwardscompatible fashion without architectural upgrades.

Naming: Intentional Naming allows mobile users to specify their "intent" and an in-network resolution mechanism matches it to available services [14]. LNA [17] and SFR [29] advocate decoupling identifiers from location and routing. IBRs share their core philosophy that binding protocols to irrelevant details limits flexibility. Our specific focus is on video sharing and decoupling it from content presentation. quFiles provides context-aware encoding using specialized file names and metadata [39]. Unfortunately, ensuring consistent names and metadata across third-party hosting sites and providers is challenging. Hence, we make a case for an algorithmic basis for IBRs.

Search engines: While search engines try to identify similar/related content, it is important to note that IBRs address an orthogonal problem. IBRs complement the discovery to enable flexible and verifiable delivery. That said, search engine providers could offer IBR-like services similar to our proposal for supporting legacy publishers (§5.4); we are not aware of products that currently offer such capabilities.

\section{CONCLUSIONS}

In this paper, we argued the case for IBRs to improve the quality of experience and accessibility of video content, given the growing heterogeneity of device and network operating conditions. The key insight underlying our work is to bind the content references to the underlying information, ignoring the details of protocols, hosts, filenames, or bits. Consumers can seamlessly choose variants from thirdparty sites that are the most appropriate fit for their devices and operating constraints, and also verify that the variants match the publisher's intent. IBRs thus allow content publishers to easily reach a wider audience without significant infrastructure costs.

We developed practical algorithms for generating IBRs, a scalable resolution backend, efficient backwards-compatible mechanisms for users to benefit from the power of IBRs, and approaches for providing additional verification. We believe that our approach has broader potential to enable new applications. For instance, users who need content in their own language or require a much larger resolution (e.g., due to vision impairments) can request that the content meet these requirements. Similarly, it can also help in challenged networks with resource constraints [41]. We also envision new IBR-enabled caches that identify alternative versions of available locally which will be more effective at reducing redundant transfers compared to URL- or data-centric caches.

\section{Acknowledgments}

We would like to thank our shepherd Jim Kurose and the anonymous reviewers for their feedback. We would also like to thank Arun Kumar for his early contributions to the implementation of the fingerprinting algorithms and Sourabh Yerfule for his help with the Android implementation. This 
work was supported in part by NSF CAREER Award (CNS0746531), NSF NetSE grant (CNS-0905134), and a gift from Cisco.

\section{REFERENCES}

[1] Caltech dataset. http://www.vision.caltech.edu/ Image_Datasets/Caltech256/.

[2] CC Web Video: Near Duplicate Web Video Dataset. http://vireo.cs.cityu.edu.hk/webvideo/.

[3] Cisco forecast. http: //blogs.cisco.com/sp/comments/cisco\_visual】 _networking \_index \_forecast \_annual $\backslash$ _update/.

[4] Greasemonkey. https://addons.mozilla.org/enUS/firefox/addon/748/.

[5] The libfooid audio fingerprinting library. http://code.google.com/p/libfooid/.

[6] The open source perceptual hash library. http://phash.org.

[7] Social recommendations are the number one way us adults discover mobile video. http://blog.telly.com/post/51153531136/socialrecommendations-are-the-number-one-way-usadults.

[8] Univ. washington image database. http://www.cs.washington.edu/research/imagedatabase/.

[9] The ycbcr color space. http://en.wikipedia.org/wiki/YCbCr.

[10] Youtube data. http://youtube-global.blogspot.com/2010/05/atfive-years-two-billion-views-per-day.html.

[11] YouTube Video Identification Beta. http://www. youtube.com/t/video_id_about.

[12] I. Sodagar. The MPEG-DASH Standard for Multimedia Streaming Over the Internet. IEEE Multimedia, 2011.

[13] A. Andoni and P. Indyk. Near-Optimal Hashing Algorithms for Approximate Nearest Neighbor in High Dimensions. In Proc. FOCS, 2006.

[14] W. Adjie-Winoto, E. Schwartz, H. Balakrishnan, and J. Lilley. The design and implementation of an intentional naming system. In SOSP, 1999.

[15] N. Ahmed, T. Natarajan, and K. R. Rao. Discrete cosine transforms. IEEE Trans. Computers, 27(1):291-301, 1974.

[16] A. Anand, A. Akella, V. Sekar, and S. Seshan. A Case for Information-Bound Referencing . In Proc. HotNets, 2010.

[17] H. Balakrishnan, K. Lakshminarayanan, S. Ratnasamy, S. Shenker, I. Stoica, and M. Walfish. A layered naming architecture for the internet. In Proc. SIGCOMM, 2004.

[18] J. S. Boreczky and L. A. Rowe. Comparison of video shot boundary detection techniques. In SPIE 2664, 1996.

[19] B. Coskun and B. Sankur. Robust video hash extraction. In EUSIPCO 2004.

[20] G. DeCandia, D. Hastorun, M. Jampani, G. Kakulapati, A. Lakshman, A. Pilchin, S. Sivasubramanian, P. Vosshall, and W. Vogels. Dynamo: amazon's highly available key-value store. In Proc. SOSP, 2007.

[21] F. Dobrian, V. Sekar, A. Awan, I. Stoica, D. A. Joseph, A. Ganjam, J. Zhan, and H. Zhang. Understanding the impact of video quality on user engagement. In Proc. SIGCOMM, 2011.

[22] F. Nah. A study on tolerable waiting time: How long are Web users willing to wait? Behaviour \& Information Technology, 23(3), May 2004.

[23] A. Ghodsi, S. Shenker, T. Koponen, A. Singla, B. Raghavan, and J. Wilcox. Information-Centric
Networking: Seeing the Forest for the Trees. In Proc. HotNets, 2011.

[24] A. Gionis, P. Indyk, and R. Motwani. Similarity search in high dimensions via hashing. In Proc. VLDB, 1999.

[25] R. M. Karp and M. O. Rabin. Efficient randomized pattern-matching algorithms. IBM J. Res. Dev., 31(2):249-260, 1987.

[26] T. Koponen, M. Chawla, B. Gon-Chun, A. Ermolinskiy, K. H. Kim, S. Shenker, and I. Stoica. A Data-Oriented (and Beyond) Network Architecture. In Proc. SIGCOMM, 2007.

[27] S. S. Krishnan and R. K. Sitaraman. Video stream quality impacts viewer behavior: inferring causality using quasi-experimental designs. In IMC, 2012.

[28] Y. Liu, F. Li, L. Guo, B. Shen, and S. Chen. A server's perspective of internet streaming delivery to mobile devices. In INFOCOM, pages 1332-1340, 2012.

[29] M. Walfish, H. Balakrishnan, and S. Shenker. Untangling the web from DNS. In Proceedings of NSDI, 2004.

[30] C. L. Novak and S. A. Shafer. Anatomy of a color histogram. In CVPR, pages 599-605, 1992.

[31] A. Oliva and A. Torralba. Building the gist of a scene: the role of global image features in recognition. Progress in brain research, 2006.

[32] H. Pucha, D. G. Andersen, and M. Kaminsky. Exploiting similarity for multi-source downloads using file handprints. In Proc. of NSDI, 2007.

[33] T. Rodrigues, F. Benevenuto, M. Cha, K. Gummadi, and V. Almeida. On Word-of-Mouth Based Discovery of the Web. In Proc. IMC, 2011.

[34] S.C. Cheung and A. Zakhor. Estimation of web video multiplicity. In Proc. SPIE Internet Imaging, 2000.

[35] L. Shang, L. Yang, F. Wang, K.-P. Chan, and X.-S. Hua. Real-time Large Scale Near-duplicate Web Video Retrieval. In Proc. ACM Multimedia, 2010.

[36] N. Tolia, M. Kaminsky, D. G. Andersen, and S. Patil. An architecture for Internet data transfer. In Proc. NSDI, 2006.

[37] M. Y. S. Uddin, H. Wang, F. Saremi, G.-J. Qi, T. F. Abdelzaher, and T. Huang. PhotoNet: A Similarity-Aware Picture Delivery Service for Situation Awareness. In Proc. RTSS, 2011.

[38] V. Jacobson, D. Smetters, J. Thornton, M. Plass, N. Briggs, and R. Braynard. Networking Named Content. In Proc. CoNEXT, 2009.

[39] K. Veeraraghavan, J. Flinn, E. B. Nightingale, , and B. Noble. quFiles: The right file at the right time. In Proc. FAST, 2010.

[40] L. von Ahn, B. Maurer, C. McMillen, D. Abraham, and M. Blum. reCAPTCHA: Human-Based Character Recognition via Web Security Measures. Science, 2008.

[41] U. Weinsberg, Q. Li, N. Taft, A. Balachandran, G. Iannaccone, V. Sekar, and S. Seshan. CARE: Content Aware Redundancy Elimination for Disaster Communications on Damaged Networks. In Proc. HotNets, 2012. 